\title{
Channel Coding for Enhanced Mobile Broadband Communication in 5G Systems
}

\author{
Heshani Gamage, Nandana Rajatheva, and Matti Latva-aho \\ Department of Communication Engineering, University of Oulu, Finland \\ E-mail: hniyagam@ee.oulu.fi,rrajathe@ee.oulu.fi,matti.latva-aho@ee.oulu.fi
}

\begin{abstract}
In this paper, candidate coding schemes are investigated for the new radio access technology (RAT) of the fifth generation (5G) mobile communication standard. Enhanced mobile broadband(eMBB) scenario of the $5 \mathrm{G}$ standard corresponding the activities in the third generation partnership project (3GPP) is considered. The coding schemes are evaluated in terms of block error rate (BLER), bit error rate (BER), computational complexity, and flexibility. These parameters comprise a suitable set to assess the performance of different services and applications. Turbo, low density parity check (LDPC), and polar codes are considered as the candidate schemes. These are investigated in terms of obtaining suitable rates, block lengths by proper design for a fair comparison. The simulations have been carried out in order to obtain BLER / BER performance for various code rates and block lengths, in additive white Gaussian noise (AWGN) channel. It can be seen from the simulations that although polar codes perform well at short block lengths, LDPC has a relatively good performance at all the block lengths and code rates. In addition, complexity of the LDPC codes is relatively low.
\end{abstract}

Index Terms - eMBB; 5G; turbo; LDPC; polar codes; 3GPP

\section{INTRODUCTION}

Services offered by cellular communication systems have evolved from the first generation( $1 \mathrm{G})$ to the fourth generation (4G) adding further enhanced services in each generation. $1 \mathrm{G}$ was only for voice calls followed by the second generation $(2 \mathrm{G})$, which added text messaging services. The third generation $(3 \mathrm{G})$ added mobile Internet services to $2 \mathrm{G}$. Currently $4 \mathrm{G}$ offers high capacity mobile multimedia service at $1 \mathrm{Gbps}$ data rate when stationary and $100 \mathrm{Mbps}$ rate when mobile, making it 250 times better than the $3 \mathrm{G}$ services. 5G New Radio (NR) is the forthcoming evolution of mobile technology expected to be in use by the year 2020 with a wide range of usability beyond the uses of 4G [1]. Performance parameters of $5 \mathrm{G}$ technologies are expected to be tens and thousands times better than in $4 \mathrm{G}$. Although performance parameters for $5 \mathrm{G}$ are not standardized yet, typical parameters may include, network capacity of ten thousands times the capacity of the current network, $10 \mathrm{Gbps}$ peak data rate with $100 \mathrm{Mbps}$ at cell edge, and less than a millisecond of network latency. Low energy consumption as well as cost are also desired. In addition $5 \mathrm{G}$ should enable machine to machine communication (M2M) at ultra low cost and ultra high reliability while supporting 10 years battery life. To facilitate these needs, spectral efficiency, signaling efficiency, bandwidth and coverage should be significantly enhanced compared to $4 \mathrm{G}$ [2].

There are two significant trends which are the main drivers of the development of 5G network technology [2]. The most critical one is the impetuous increase in demand for wireless broadband services needing faster, higher-capacity networks that can deliver video and other content-rich services. This need is driven by the growth in the video traffic and new applications such as full high definition (FHD), live video broadcast and virtual reality, requiring ultra broadband services. This was the primary drive of the $4 \mathrm{G}$ as well and now it needs 1000 times more capacity and data rates. The other requirement of $5 \mathrm{G}$ is the rapidly evolving area of Internet of things (IoT), needing a massive connectivity of devices with ultrareliable, ultra-low-latency connectivity over Internet Protocol (IP). This includes a variety of applications, such as vehicle-tovehicle and vehicle-to-infrastructure transportation systems for autonomous driving and driver assistance, industrial automation and utility applications, wireless health services, virtual and augmented reality services, some smart city applications, and smart homes. These applications demand to facilitate communication between machines instead of humans, hence requirements of these applications significantly differ from mobile broadband services as they require low latency and high reliability at a low data rate. In addition power consumption and cost should also be low in IoT applications.

Based on different user requirements, new radio access technology for $5 \mathrm{G}$ systems includes three scenarios; namely 1. eMBB 2. Ultra reliable low latency communications (URLLC) and 3. Massive machine type communications (mMTC). According to its usage, URLLC and mMTC are latency sensitive and need high reliable communication where as eMBB demands higher data rates and capacities. From these three scenarios, eMBB remains the most critical as the ongoing growth of users demanding the eMBB services proves to be strong and profitable. Requirement of the eMBB scenario is to support a much wider range of code rates, code lengths and modulation orders than the 4G Long Term Evolution (LTE). In the current assumption, eMBB code lengths range from 100 to 8000 bits (optionally 12,000-64000 bits) and code rate ranges from $1 / 5$ to $8 / 9$.

In order to cater for the requirements of eMBB, $5 \mathrm{G}$ needs to achieve significant enhancements in spectral efficiency, signaling efficiency, bandwidth and coverage compared to $4 \mathrm{G}$. Achieving high spectral efficiency will lower the cost per bit. In order to achieve high spectral efficiency, channel coding 
and modulation plays significant roles in the physical layer. There are key technologies [3] expected to enable the performance levels of eMBB. They are, extreme densification and offloading, increasing bandwidth with the usage of millimeterwave (mmWave) spectrum and massive MIMO technologies. In addition, new physical layer and MAC search for a number of new possibilities such as novel wave forms, multiple access schemes, and modulation methods as a new versatile radio access technology. Channel coding plays an important role in order to have a fast and error free communication since data transmission occurs in an imperfect channel environment. The selected channel code must have an excellent BLER performance in a specific range of block lengths and code rates. Low computation complexity, low latency, low cost and higher flexibility are desired for the coding scheme. Furthermore reduced energy per bit and improved area efficiency are required to support higher data rates.

Tail biting convolutional codes (TBCC), turbo codes, LDPC codes and polar codes are the competing coding schemes considered as the candidates for 5G. However, TBCC is not considered for the eMBB scenario since it has a poor performance in large block lengths and low code rates. In this paper, candidate error correction coding schemes for $5 \mathrm{G}$ eMBB scenario are reviewed and their performances are evaluated.

The rest of this paper is organized as follows. In section II some of the existing work of turbo, LDPC and polar coding schemes is discussed. In Section III, various performance aspects of the candidate coding schemes, such as computational complexity and flexibility are discussed along with encoding and decoding methods. In Section IV parameters and methods used in simulations are explained. BLER and BER simulations results are given in Section $\mathrm{V}$, followed by conclusions in Section VI.

\section{RELATED WORK}

Although many coding schemes with capacity achieving performance at large block lengths are available, many of those do not show consistent good performance in a wide range of block lengths and code rates as the eMBB scenario demands. But turbo, LDPC and polar codes show promising BLER performance in a wide range of coding rates and code lengths; hence, are being considered for $5 \mathrm{G}$ physical layer.

Introduced in [4], turbo code encoder is built using a parallel concatenation of two recursive systematic convolutional codes and the associated decoder, using a feedback decoding rule. Due to the low error probability performance within a $1 \mathrm{~dB}$ fraction from the the Shannon limit, turbo codes are being used in a variety of applications, such as deep space communications, 3G/4G mobile communication in Universal Mobile Telecommunications System (UMTS) and LTE standards and Digital Video Broadcasting (DVB). Although it is being used in $3 \mathrm{G}$ and $4 \mathrm{G}$, it may not satisfy the performance requirements of eMBB for all the code rates and block lengths as the implementation complexity is too high for higher data rates. In addition, an error floor is observed in turbo code BER. In [5, Fig. 8.18], the error floor is at BER of $10^{-6}$.

LDPC codes were originally invented and published in [6] in 1962, they were not in use until it was rediscovered by
Mackay [7] in 1997. He showed that the empirical performance of LDPC codes can approach the Shannon limit similar to turbo codes or even better. LDPC codes are linear codes and as the name suggests, has a sparse parity check matrix consisting low density ' $1{ }^{\prime} \mathrm{s}$. Due to the sparsity of the parity check matrix, LDPC codes have relatively simple and practical decoding algorithms. Decoding is done by iterative belief propagation algorithms, which estimates bit values and parity check values in bit and check nodes respectively, and passing values between them in each iteration. The accuracy of the estimates will be improved in each iteration and the number of iterations is decided based on the requirement of the application. Tradeoffs are possible between the bit error performance, latency and the complexity. Modern LDPC decoders work with soft decision algorithms, which further enhance the decoder gain. Due to their excellent ability to achieve theoretical limits of channel capacity, LDPC codes are currently being used in many communication systems such as DVB-S2, 802.11n (WiFi allowing MIMO) and 802.16e (Mobile WiMAX) etc.

Turbo codes and LDPC codes were competing against each other in various use cases and applications, as both of these coding schemes show good performance. Turbo codes generally have a low encoding complexity and high decoding complexity whereas LDPC codes have a high encoding complexity but low decoding complexity.

Polar codes were introduced by Arikan [8] in 2009 and they are the first provably capacity-achieving codes with low encoding and decoding complexities. Polar codes outperform turbo codes in large block lengths, while no error floor is present. The concept behind channel polarization in polar codes is transforming $N$ copies (transmissions) of the channel (e.g., say AWGN channel) with a symmetric capacity of $I(W)$ into extreme channels of capacity close to one or zero. Out of $N$ channels, $I(W)$ fraction will become perfect channels and $1-I(W)$ fraction of channels will become completely noisy channels. Then, the information bits are sent only through good channels while inputs to other channels are made frozen into one or zero. The amount of channel polarization increases with the block length. Some of the issues with polar codes are, that the code design is channel dependent, hence not versatile for mobile fading channels and the limited application experience due to its immaturity. Polar codes have better energy-efficiency for large block lengths than other codes.

\section{CANDidAte Coding Schemes}

\section{A. Turbo codes}

3GPP LTE standard specification parameters [9] are used for the simulations and the discussions of turbo codes in this paper. Each convolutional turbo code encoder outputs two streams, one systematic stream and one parity stream. Input information bits to the second encoder is fed after interleaving the input bit stream. For iterative decoding of the parallel concatenated encoding scheme, the turbo decoder uses a MAX-Log-MAP algorithm as the constituent decoder component. The internal interleaver of the decoder is identical to the one the encoder uses. 
Table I: Number of equivalent additions per operation [11].

\begin{tabular}{|l|c|}
\hline Operation & $\begin{array}{c}\text { Number of Equivalent } \\
\text { additions per operation }\end{array}$ \\
\hline Addition,Subtraction & 1 \\
\hline \pm 1 Multiplication, Division & 1 \\
\hline Comparison & 2 \\
\hline Maximum, Minimum & 1 \\
\hline Parallel list & 1 \\
\hline
\end{tabular}

Since there are three output streams; a systematic bit stream and two parity streams in the encoder, coding rate for this LTE system is $1 / 3$. Other higher rates are achieved through puncturing of some parity bits. Assuming the decoder has a memory length of $M$ bits for the component code and $I$ number of iterations, then turbo codes has a complexity of 16.I.R.N. $2^{M}$ additions and 8.I.R.N. ${ }^{M}$ logical operations. [10] Complexity is expressed in terms of equivalent additions per operation. Number of equivalent additions for different operations are stated in Table I.

\section{B. LDPC codes}

Quasi-Cyclic LDPC (QC-LDPC) codes are used for the simulations and discussions throughout this paper. A QCLDPC matrix is characterized by the parity check matrix $\mathrm{H}$. It consists of small square blocks which are the zero matrix or circulant permutation matrices. An advantage of QC-LDPC code is that it supports variable code lengths which can be easily obtained by adjusting the circulant permutation matrices in H. Sum product algorithm (SPA) is the optimal decoder for LDPC, and min-sum algorithm is a sub optimal algorithm with reduced computational complexity.

While QC-LDPC codes allow reasonable flexibility in code length, puncturing is used to achieve rate-adaptive codes. Assuming $d_{v}$ and $d_{c}$ as the average variable and check degrees of the LDPC parity check matrix respectively and $\mathrm{P}$ as the number of parity bits, min-sum LDPC decoder has $I .\left(2 N d_{v}+2 P\right)$ additions and $I .\left(2 d_{c}-1\right) . P$ MAX process/comparisons [10]. Offset min-sum (OMS) decoder is a reduced complexity version of min-sum decoder where algorithm converges in a smaller number of iterations and it is used as the decoder for the simulations in this paper.

\section{Polar codes}

Polar code construction can be done recursively via Kronecker products and have an encoding complexity of $O(n \log n)$. Complexity of decoding is also the same via successive cancellation (SC) decoding. However, the SC decoder itself is not sufficient to achieve competitive performance as other coding schemes. Hence list decoding is incorporated into SC decoder which results in the SC list (SCL) decoder [13]. In addition a cyclic redundancy check bits (CRC) are used to further enhance the code performance at an expense of increased complexity.
Table II: Parameters for simulations.

\begin{tabular}{|l|l|l|l|}
\hline Parameters & Turbo & LDPC & Polar \\
\hline Channel & AWGN & QAM \\
\hline Modulation & Code lengths & $128,512,1024,2048$ & 8 \\
\hline Code rates & $1 / 3,1 / 2,2 / 3$ & NA & $\begin{array}{l}\text { SC, CRCA- } \\
\text { SCL, L=8 }\end{array}$ \\
\hline CRC & NA & $\begin{array}{l}\text { Offset-min- } \\
\text { sum }\end{array}$ & max-log-MAP
\end{tabular}

Table III: Decoding complexity of coding schemes.

\begin{tabular}{|c|l|l|l|l|l|l|l|}
\hline \multirow{2}{*}{$\begin{array}{l}\text { Block } \\
\text { length }\end{array}$} & $\begin{array}{l}\text { Coding } \\
\text { Scheme }\end{array}$ & \multicolumn{3}{|c|}{ Complexity $\left(\times 10^{3}\right)$} & \multicolumn{3}{|c|}{ Percentage } \\
\cline { 2 - 8 } 128 & Turbo & 65.5 & 98.3 & 131.1 & $100 \%$ & $100 \%$ & $100 \%$ \\
\cline { 2 - 8 } & LDPC & 66.0 & 57.2 & 48.5 & $100.7 \%$ & $58 \%$ & $37 \%$ \\
\cline { 2 - 8 } & $\begin{array}{l}\text { Polar } \\
\text { SC }\end{array}$ & 1.0 & 1.0 & 1.0 & $1.5 \%$ & $1.0 \%$ & $0.8 \%$ \\
\cline { 2 - 8 } & $\begin{array}{l}\text { Polar } \\
\text { SCL }\end{array}$ & 11.0 & 11.0 & 11.0 & $16.8 \%$ & $11.2 \%$ & $8.4 \%$ \\
\hline \multirow{3}{*}{2048} & Turbo & 1048.6 & 1572.9 & 2097.9 & $100 \%$ & $100 \%$ & $100 \%$ \\
\cline { 2 - 8 } & LDPC & 1056 & 916 & 776 & $100.7 \%$ & $58.2 \%$ & $37.0 \%$ \\
\cline { 2 - 8 } & $\begin{array}{l}\text { Polar } \\
\text { SC }\end{array}$ & 24.6 & 24.6 & 24.6 & $2.3 \%$ & $1.6 \%$ & $1.2 \%$ \\
\cline { 2 - 8 } & $\begin{array}{l}\text { Polar } \\
\text { SCL }\end{array}$ & 245.5 & 245.5 & 245.5 & 23.4 & $15.6 \%$ & $11.7 \%$ \\
\hline
\end{tabular}

Construction of the polar codes requires knowledge about specific channel conditions, hence not versatile. While different code rates can be achieved by changing the number of frozen bits in polar codes, a puncturing scheme is needed to obtain some code lengths. SC decoder has a complexity of only $N \log _{2} N$ additions where as SCL decoder with list size of $L$ has a complexity of L.N. $\log _{2} N+(N-P) \cdot L \cdot \log _{2} L$ additions [10].

\section{Simulations}

The candidate coding schemes are simulated with Quadrature amplitude modulation (QAM). An AWGN channel is considered with Channel state information (CSI). Coding parameters are given in Table II and the performance is evaluated in terms of BLER and BER.

For turbo codes, the LTE-Advanced encoder with two 8state constituent encoders and a block interleaver is used. At the receiver, the scaled MAX-Log-MAP decoder with scaling factor of 7 is used with 8 iterations. The code rate of original turbo code is $1 / 3$ and higher rate codes are obtained through puncturing. Parity check matrices specified in IEEE $802.11 \mathrm{n}$ are used for encoding in LDPC simulations. The OMS decoder with 0.3 as the offset value and 50 iterations is used at the receiver. Different code lengths are obtained by changing the size of base matrix of the parity check matrix. 

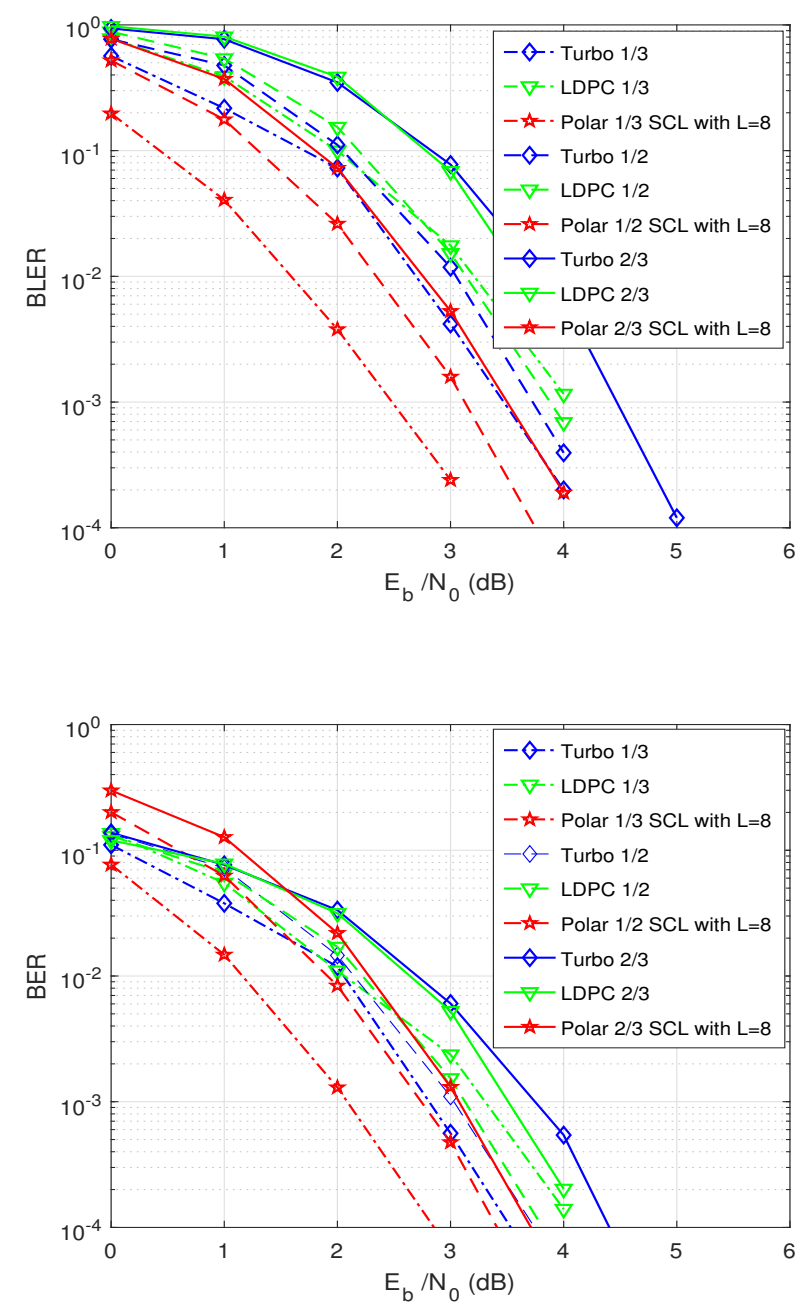

Figure 1: BLER and BER performance of coding schemes at a coded block length of 128 bits and QAM modulation for different code rates.

Polar codes construction is based on construction for AWGN channel as suggested in [14]. The coding scheme performance is simulated CRC aided-SCL decoder (CRCA-SCL) with list size of 8 and $8 \mathrm{CRC}$ bits for all the code rates and lengths.

Algorithmic complexity of the decoders used for each coding scheme is obtained for block lengths 128 and 2048 (Table III). This also shows the percentage of complexity with respect to turbo code in the percentage column.

\section{Simulation Results}

In Fig. 1 to 4, BLER and BER of the coding schemes are plotted against the energy per bit to noise power spectral
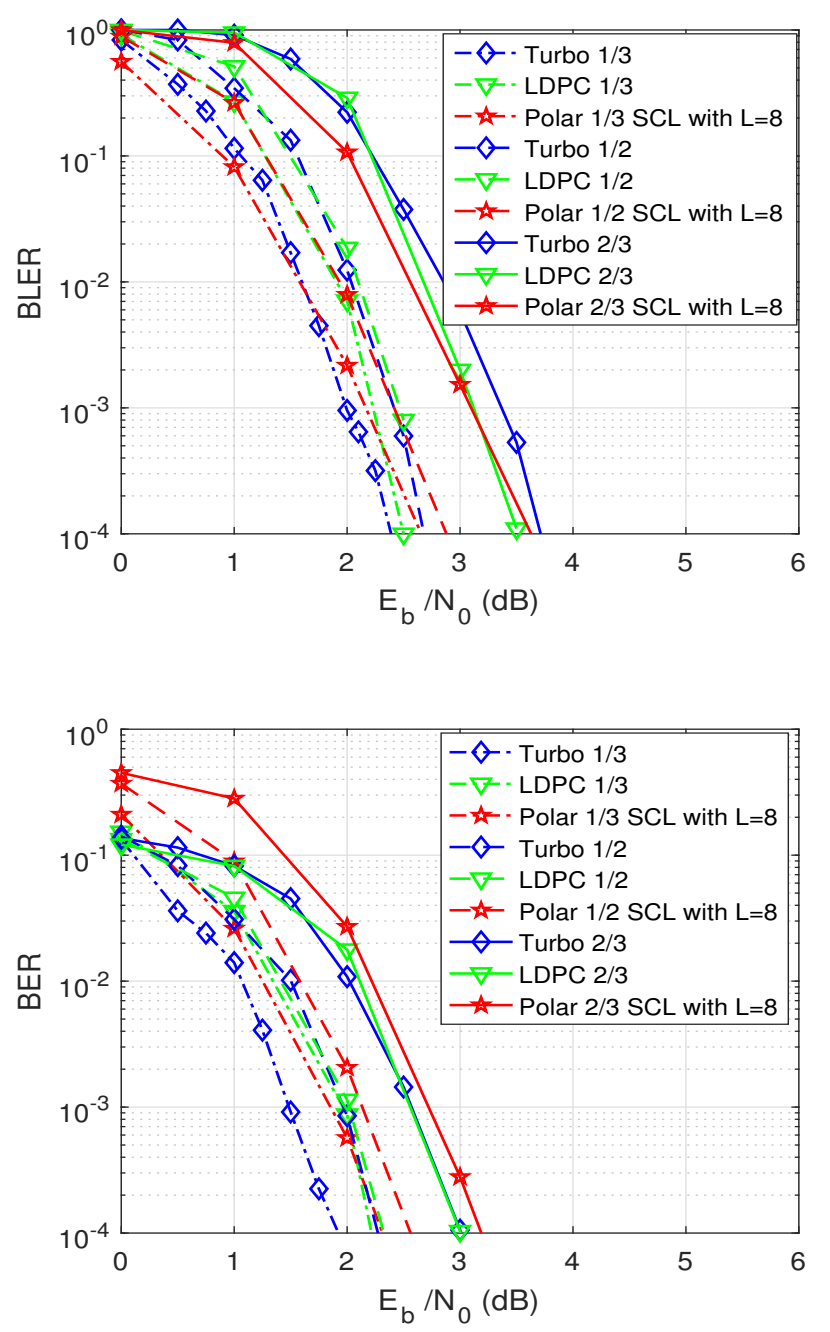

Figure 2: BLER and BER performance of coding schemes at a coded block length of 512 bits and QAM modulation for different code rates.

density ratio $\left(E_{b} / N_{0}\right)$ for Turbo, LDPC, and Polar codes for different block lengths and coding schemes.

It can be seen that at the block-length of 128 bits (Fig. 1), polar codes with CRCA-SCL decoder outperforms turbo and LDPC codes for all the 3 rates. As the block length increases, LDPC and Turbo coding scheme performance comes close to the performance of the polar codes with CRCA-SCL decoder. For example, at the block length of 512 bits (Fig. 2), polar codes with CRCA-SCL decoder has similar performance as Turbo for rate $1 / 3$ in BLER. But turbo outperforms polar codes in BER at this block length and rate.

When the block length increases as in Fig. 3 - 4 , turbo codes outperform polar codes with CRCA-SCL decoder and LDPC 

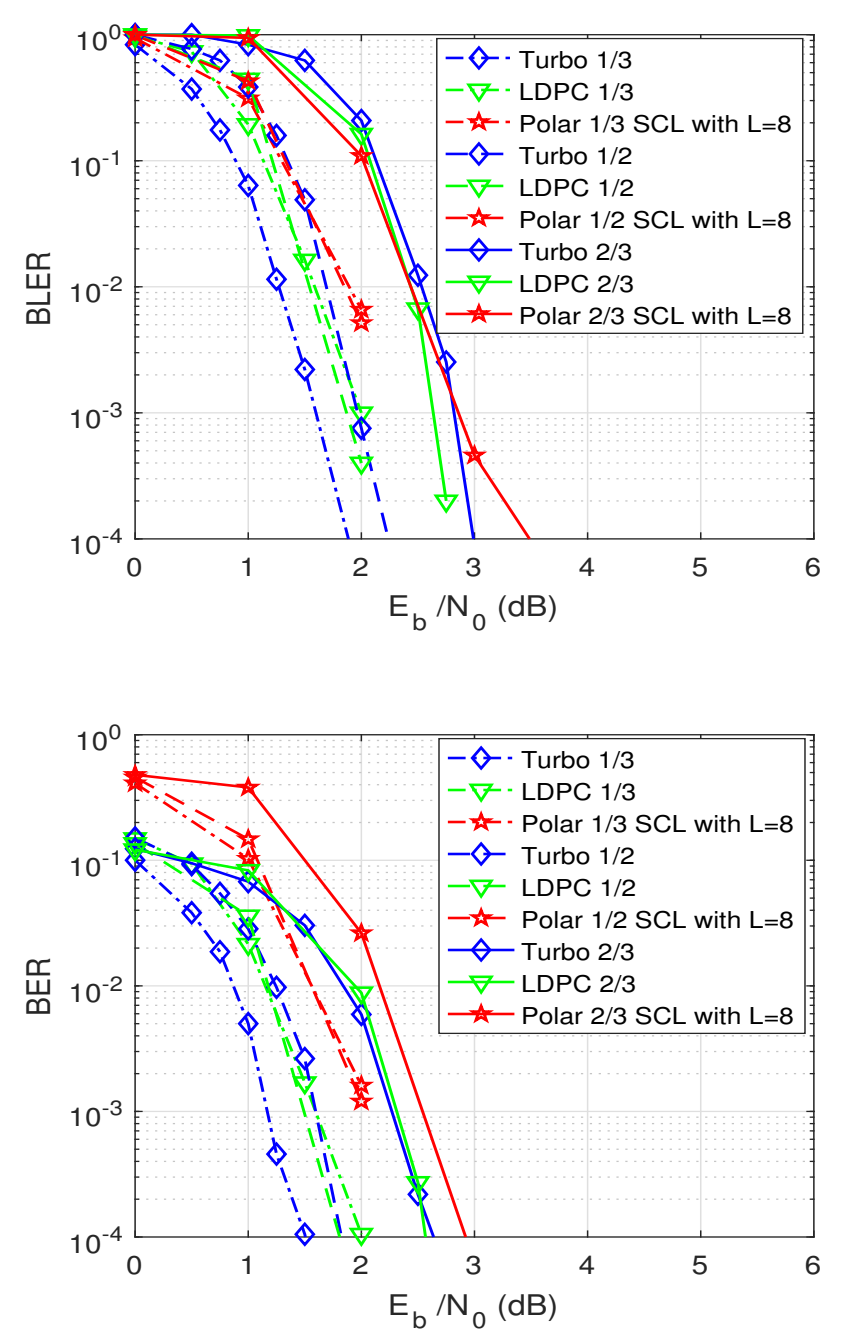

Figure 3: BLER and BER performance of coding schemes at a coded block length of 1024 bits and QAM modulation for different code rates.

for rate $1 / 3$. For rates $1 / 2$ and $2 / 3 \mathrm{LDPC}$ has better performance than polar and turbo. It should be noted that LDPC codes performs relatively well, even without the use of CRC bits. hence this performance can be further enhanced by adding $\mathrm{CRC}$ at a cost of increased complexity.

In terms of algorithmic complexity, as computed in Table III, Turbo and LDPC codes shows similar complexity in rate $1 / 3$. But for all the other rates turbo codes has relatively higher complexity. SC decoder of Polar codes has the lowest complexity for all rates and SCL decoder with list size of 8 has about 10 times the complexity of SC decoder. It should be noted that these complexities are based on the assumptions in the Table I and actual implementation complexity may differ
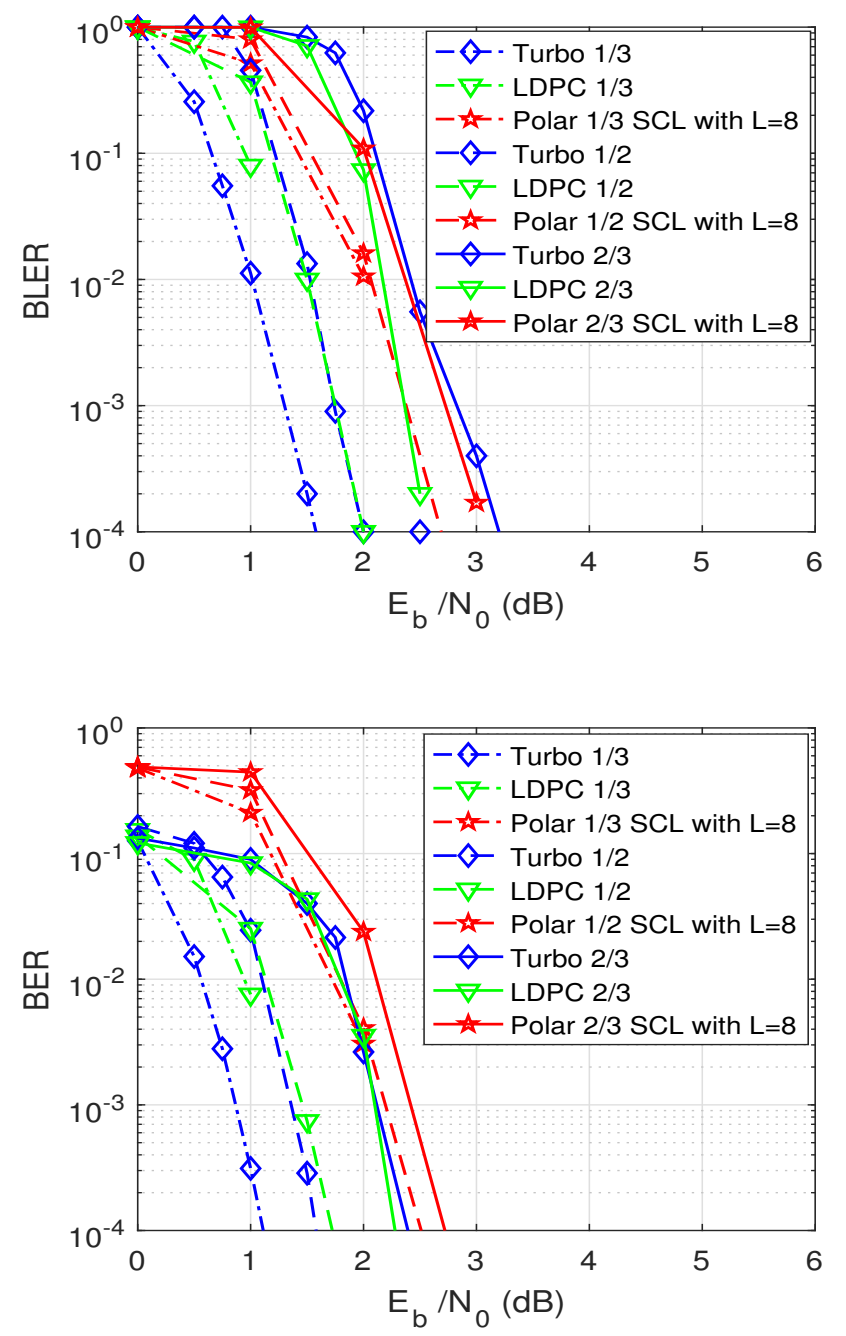

Figure 4: BLER and BER performance of coding schemes at a coded block length of 2048 bits and QAM modulation for different code rates.

in practical scenarios.

\section{CONCLUSION}

In this paper, several performance aspects of candidate coding schemes for 5G NR are analyzed. For short block lengths around 124 bits, polar codes with CRCA-SCL decoder have better performance than Turbo and LDPC codes. However, LDPC codes exhibit relatively good performance in all the coding rates and block lengths. Furthermore, it should be noted that LDPC codes show this performance without the aid of CRC. Hence, LDPC performance can be further enhanced by using a CRC. 
On the other hand, polar codes have the benefit of not having an error floor compared to LDPC and turbo, both of which have error floors. However, to achieve the optimal performance of polar codes, code construction should be done based on the channel, hence polar codes are not yet versatile. Further research should be conducted to achieve channel independent code design.

Although SC decoder of polar codes shows the lowest complexity, CRC aided SCL decoder exceeds the complexity of LDPC codes and turbo codes. Actual costs of SCL decoder is uncertain due to lack of implementations.

There are many other factors to be considered when choosing a coding scheme, such as latency for encoding and decoding, energy efficiency, and area efficiency. In current implementations, LDPC codes shows relatively good performance in both area and energy efficiency. Turbo codes consumes highest energy per bit and very low area efficiency. Turbo codes has good energy efficiency and low area efficiency. Exact area and energy efficiency of SCL decoder of polar codes are not known yet due to lack of implementations.

In November 2016, 3GPP radio access network (RAN) \#87 meeting, LDPC codes were agreed to be adopted for both uplink and downlink eMBB data channels [15]. Furthermore, polar coding is to be adopted for both uplink and downlink control information channels [15].

\section{REFERENCES}

[1] N. Rajatheva, S. Suyama, W. Zirwas, L. Thiele, G. Fodor, A. Tolli, E. De Carvalho, J. H. Sorensen, "Massive multiple input multiple outpu (MIMO) systems," 5G Mobile and Wireless Communications Technology, Afif Osseiran, J. F. Monserrat and P. Marsch Eds., Cambridge University Press, Chapter 8, pp. 208-247, June 2016.

[2] "The Road to 5G: Drivers, Applications, Requirements and Technical Development," Global Mobile Suppliers Association(GSA) Executive Report, November 2015.
[3] J.G. Andrews, S. Buzzi, W. Choi, S.V. Hanly, A. Lozano, A.C.K. Soong, and J.C. Zhang, "What Will 5G Be?," IEEE Journal on Selected Areas in Communications, vol. 32, no. 6, pp.1065-1082, June 2014.

[4] C. Berrou, A. Glavieux and P. Thitimajshima, "Near Shannon limit error-correcting coding and decoding: Turbo-codes. 1," IEEE International Conference on Communications, 1993.

[5] A. Goldsmith, "Wireless communications" Cambridge university press, 2005.

[6] R.G. Gallarger, "Low Density Parity Check codes," IRE Transactions on Information Theory, 1962.

[7] D.J.C. MacKay and R.M. Neal, "Near Shannon limit performance of low density parity check codes," Electronic Letters, Vol. 33, No. 6, March 1997.

[8] E. Arikan, "Channel Polarization: A Method for Constructing CapacityAchieving Codes for Symmetric Binary-Input Memoryless Channels," IEEE Transactions on Information Theory, vol. 55, no. 7, pp.30513073, July 2009.

[9] ETSI, "Evolved Universal Terrestrial Radio Access (E-UTRA) Multiplexing and Channel Coding" 3GPP Technical specification, version 12.6.0, Release 12

[10] M. Sybis, K. Wesolowski, K. Jayasinghe, V. Venkatasubramanian, and V. Vukadionovic,"Channel coding for ultra-reliable low-latency communication for 5G systems," IEEE Vehicular Technology Conference, 2016.

[11] P. H. and Y. Wu, "On the complexity of turbo decoding algorithms," IEEE Vehicular Technology Conference, vol. 2, pp.1439-1443, May 2001.

[12] C. Studer, C. Benkeser, S. Belfanti, and Q. Huang, "Design and Implementation of a Parallel Turbo-Decoder ASIC for 3GPP-LTE," IEEE Journal of Solid-State Circuits 2011.

[13] I. Tal and A. Vardy, "List Decoding of Polar Codes," IEEE Transactions on Information Theory, vol. 61, no. 5, pp.2213-2226, May 2015.

[14] H. Vangala, E. Viterbo and Y. Hong, "A Comparative Study of Polar Code Constructions for the AWGN Channel," IEEE Transactions on Information Theory, vol. 61, no. 5, pp.2213-2226, Jan. 2015.

[15] Session Chairman (Nokia), "Chairman's Notes of Agenda Item 7.1.5 Channel coding and modulation," 3GPP TSG RAN WG1 Meeting 87, R1-1613710, Reno, USA, November 2016. 